\title{
Research on Defects Positioning Method of Barrel Based on $L(0,2)$ Mode
}

\author{
GAO Wang ${ }^{1, a}$, ZHANG Jin ${ }^{2, b}$ \\ 1.Postgraduate Management Team, Army Officer Academy, Hefei 230031, China \\ 2.Army Officer Academy, Hefei 230031, China \\ a176583166@qq.com, ${ }^{b} 270871979 @ q q . c o m$
}

Keywords:ultrasonic guided wave; defect; gun barrel; longitudinal mode; propagation characteristics

\begin{abstract}
Guns, especially new type guns play an important role in modern warfare. As an important part of gun, the barrels' damage detection is of great significance. After studying and comparing the characteristics of $\mathrm{L}$ mode and $\mathrm{T}$ mode ultrasonic guided waves, this paper puts forward a nondestructive detection method based on $\mathrm{L}(0,2)$ mode guided wave. By using finite element software-Comsol, this paper simulates the guided wave propagation characteristics when asymmetric defects exist on the inner surface of gun barrel. The research and simulation results indicate that this method can be used to recognize guided wave mode and detect defects. It is hoped that this paper can provide some instructions to future researches on barrel detection.
\end{abstract}

\section{Introduction}

As an emerging non-destructive testing method, ultrasonic guided wave detection technology has been widely applied in pipeline inspection, complex structures detection and other fields owe to its unparalleled advantage. Lin Wu studied the propagation characteristics of ultrasonic guided wave in the rail and provided a theoretical basis for on-line rail detection technology [1]. Xuewei-Sun deeply studied the guided wave propagation mechanism of complex structures, including thick beams, thick-walled pressure vessels and variable cross-section structure (high-speed rail axle), and studied the effects on propagation characteristics of guided waves caused by different defect types in different structures[2].

Researches on the application of the guided wave technology in barrel detection are quite few. Changes of parameters and complex process make defect types different. On the basis of previous researches, this paper puts forward a detection method based on $\mathrm{L}(0,2)$ mode guided wave, which can effectively detect barrel defects and improve the detection efficiency.

\section{Type Ultrasonic Guided Wave Nondestructive Testing Methods of Barrels}

Ultrasound guided wave propagation in the cylinder structure [3] has its conditions, the boundary conditions are as follows:

$$
\sigma_{\mathrm{rr}}=\sigma_{\mathrm{rz}}=\sigma_{\mathrm{r} \theta}
$$

In the above formula, a and b represents the inner and outer radius of the cylinder respectively.

This paper discusses this question by using cylindrical coordinates and assumes the three displacement components are the following during the process of propagation:

$$
\left\{\begin{array}{l}
u_{r}=U_{r}(r) \cos n \theta \cos (\omega t+\varepsilon z) \\
u_{\theta}=U_{\theta}(r) \cos n \theta \cos (\omega t+\varepsilon z) \\
u_{z}=U_{z}(r) \cos n \theta \cos (\omega t+\varepsilon z)
\end{array}\right.
$$

In the above formula, $n$ represents the order of the circumferential direction, $\varepsilon$ is the wave number, $\omega$ is the circular frequency, $U_{r}(r), U_{\theta}(r), U_{z}(r)$ are the corresponding displacement amplitudes.

This paper uses Helmholtz to decompose the displacement vectors of the particle into scalar potential function $\phi$ and vector potential function $\mathrm{H}$ : 


$$
\left\{\begin{array}{l}
u=\nabla \phi+\nabla \times H \\
\nabla \cdot H=0
\end{array}\right.
$$

To solve the ultrasonic guided wave of displacement field is to solve the following dispersion equation in the final analysis:

$\mathrm{C}_{\mathrm{ij}}$ represents the diameter size.

$$
D=\left|\begin{array}{cccc}
C_{11} & C_{12} & \cdots & C_{16} \\
C_{21} & C_{22} & \cdots & C_{26} \\
\vdots & \vdots & \ddots & \vdots \\
C_{i j} & C_{62} & \cdots & C_{66}
\end{array}\right|=0
$$

When $n=0$, the modal of ultrasonic is axial symmetry, thus the dispersion equation can be decomposed into two sub-plot determinant.

$$
\text { And } D_{1}=\left|\begin{array}{llll}
C_{11} & C_{12} & C_{14} & C_{15} \\
C_{31} & C_{32} & C_{34} & C_{35} \\
C_{41} & C_{42} & C_{44} & C_{45} \\
C_{61} & C_{62} & C_{64} & C_{65}
\end{array}\right| ; \quad D_{2}=\left|\begin{array}{ll}
C_{23} & C_{26} \\
C_{53} & C_{56}
\end{array}\right| \text {. }
$$

$$
D=D_{1} \cdot D_{2}=0
$$

When $\mathrm{D}_{1}=0, \mathrm{D}_{2}=0$, the results are respectively corresponding to the longitudinal mode of $\mathrm{L}(0, \mathrm{~m})$ and the torsional mode $\mathrm{T}(0, \mathrm{~m})$ of the axial symmetry.

When the parameters are put into this formula, the phase velocity and group velocity frequency dispersion curves of L mode guided waves are shown as Fig.1.

To avoid multiple modes guided waves of the barrels occur under specific excitation frequencies and lower the identification difficulty, this paper selects the excitation frequency of $250 \mathrm{KHz}$. Theoretically, $L(0,2)$ mode guided waves can be excited in the barrel for barrel detection. From the above figure, this paper derives that when the excitation frequency is $250 \mathrm{KHz}$, the group velocity of $\mathrm{L}(0,20)$ mode guided wave $\mathrm{Cg} 1$ is $5388.5 \mathrm{~m} / \mathrm{s}$, the group velocity of $\mathrm{L}(0,1)$ mode guided wave Cg2 is $2478.84 \mathrm{~m} / \mathrm{s}$.

\section{Barrel Guided Wave Finite Element Modeling}

Here is an example of a certain type of weapon barrel which is in service. Its inner radius $a=49 \mathrm{~mm}$, outer radius $b=50 \mathrm{~mm}$. The material parameters are as follows: Young's modulus $\mathrm{E}$ is $207 \times 109 \mathrm{~Pa}$, Poisson's ratio $\mu=0.3$, the mass density $\rho=7800 \mathrm{~kg} / \mathrm{m} 3$.

\section{A. Modeling}

This paper uses an equivalent method and exerts axial force on the barrel surface directly. Instead of ultrasonic guided wave vibration, we observe the displacement of the particle directly. Through this method, simulation requirements can be greatly reduced and clear experimental results can also be obtained [4].

We use the geometric drawing module of Comsol for barrel modeling, model results obtained are shown in Fig.2.

B. Set Boundary Conditions for Solving

5 single-tone sinusoidal signal cycles by Hanning window is applied at one end of the barrel along the axial direction of the modulation of the barrel, instead of the piezoelectric effect. Expression of applied force as follows:

$$
\mathrm{F}=100 * \sin (2 * \mathrm{pi} * \mathrm{f} * \mathrm{t}) *(0.5-0.5 * \cos (2 * \mathrm{pi} * \mathrm{f} * \mathrm{t} / \mathrm{n})) *(\mathrm{t}<\mathrm{n} / \mathrm{f})
$$

In the above formula, $\mathrm{f}=250 \mathrm{KHz}, \mathrm{n}=5$. The specific waveform is shown in Fig.3.

In solving the calculation, Comsol has a high requirement on mesh generation. If the mesh is too large, it may cause large errors. If the mesh is too small, it will take enormous time and energy and reduce the computational efficiency. In this research, we had several simulation and repeated verification, and ultimately determine the size of the mesh as 1/100 of the wavelength. On this basis, 
we need to refine the mesh in the defective parts, which may be helpful in further study on defects' influence on guided wave propagation.
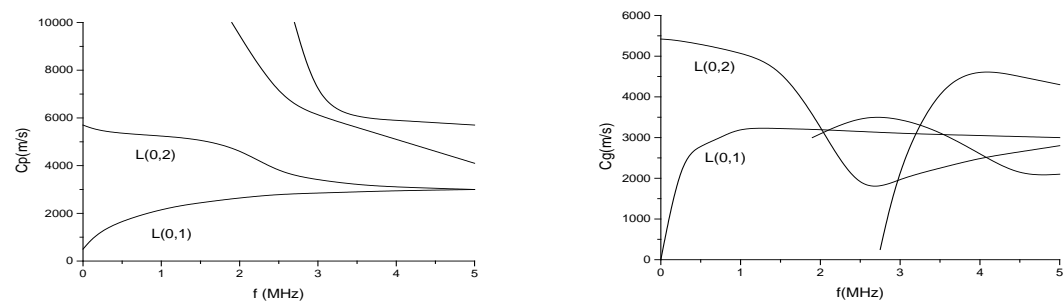

Figure 1. Dispersion curves:(a)phase velocity frequency dispersion curve(b)group velocity frequency dispersion curve
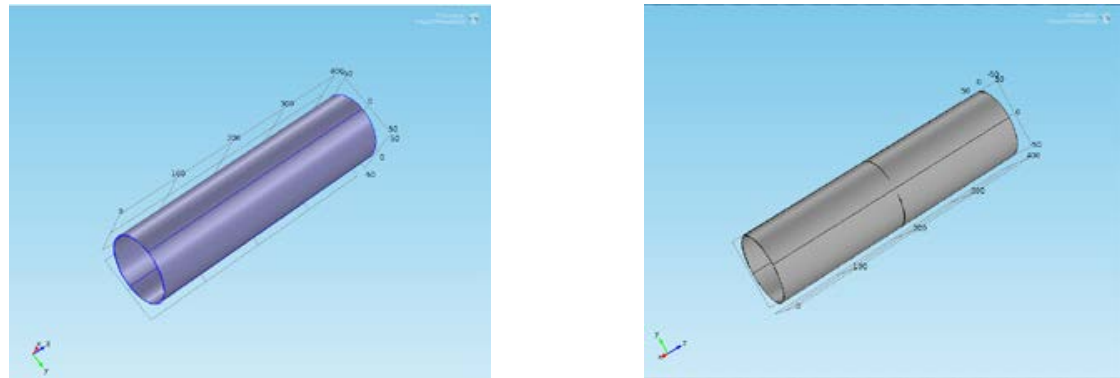

Figure 2. Barrel models: (a)barrel model without defects (b)barrel model with defects

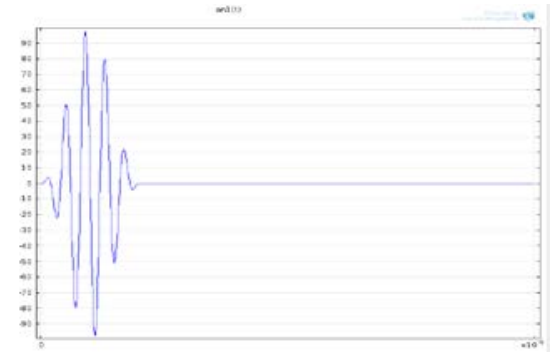

Figure 3. Excitation signal

Before computing, we must firstly set the time step and ensure that guided wave propagation is in one mesh within the time step:

$$
\mathrm{V}^{*} \mathrm{t}<\mathrm{n}
$$

In the above inequality, " $\mathrm{t”}$ is the time step and " $\mathrm{n}$ " is cell size.

In this way, accuracy can be improved and thus simulation accuracy can also be ensured. So we set the time step as 0.05us.

\section{Barrel Guided Wave Propagation Characteristics Simulation}

\section{A. Propagation Characteristics Simulation of Two Types of Guided waves}

Ultrasonic guided waves are divided into longitudinal modes, torsional mode and bending mode. The former two modals are axial symmetric and the latter is non-axial symmetric. Due to the nonaxial symmetry of the bending mode, modal conversion will occur in the dissemination process when using guided wave to detect gun barrel, which will increase the difficulty of detection. So in common detection, people usually choose longitudinal modes and torsional modes. And introduction of these two modes are as follows:

1.longitudinal modes

Using Comsol simulation L $(0,2)$ mode guided wave propagation process is shown in the Fig.4.

In the right of this figure, we use a color bar with different colors to represent the magnitude of displacement, thus displacement magnitude in different locations can be clearly and directly shown. 
In the process of $\mathrm{L}(0,2)$ mode guided wave propagation, the displacements of the axial (Zdirection), radial (R direction), the circumferential (PHI direction) are in the Fig.5.

In the above figure, the solid blue line is the axial displacement of $L(0,2)$ mode guided wave, the red dotted line represents radial displacement of $\mathrm{L}(0,2)$ mode guided wave, the yellow polyline represents circumferential displacement of $\mathrm{L}(0,2)$ mode guided wave, circumferential displacement almost equals to zero. In the propagation process, $\mathrm{L}(0,2)$ mode guided wave only has the axial and radial displacement, but has no circumferential displacement, which is consistent with the theoretical results [5]. And radial displacement is relatively smaller than axial displacement and radial energy leakage is less. Thus it can spread in a far distance, which is in line with the one-time and long-range detection requirement of guided wave testing requirements and greatly improves the detection efficiency. :

\section{2. the torsion mode}

The simulated $\mathrm{T}(0,1)$ mode guided wave propagation process is shown in Fig.6

The figure above shows the propagation process of $\mathrm{T}(0,1)$ torsional modes. However, in the actual testing, since $\mathrm{T}(0,1)$ mode waveguide excitation needs piezoelectric sheet distributed along the entire circumference of the barrel, and requires stimulate the tangential direction, it is not easy to achieve. Therefore this kind of mode is generally not applied in barrel detection.

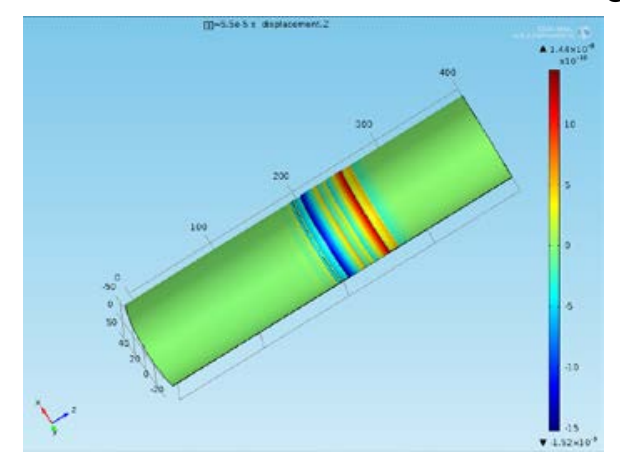

Fig.4. Propagation of L(0,2) mode guided wave

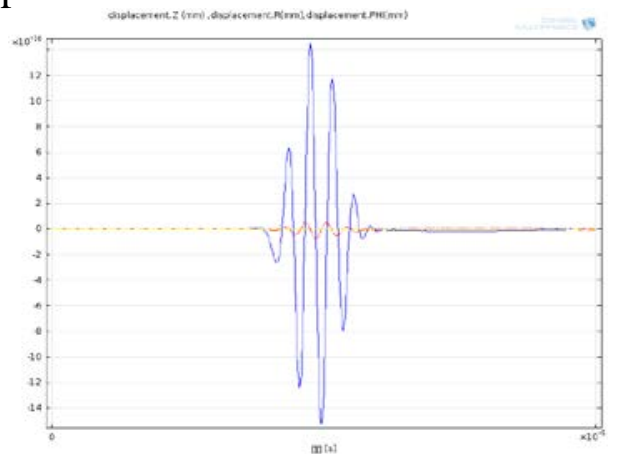

Fig.5 Each displacement of L(0,2) mode guide wave

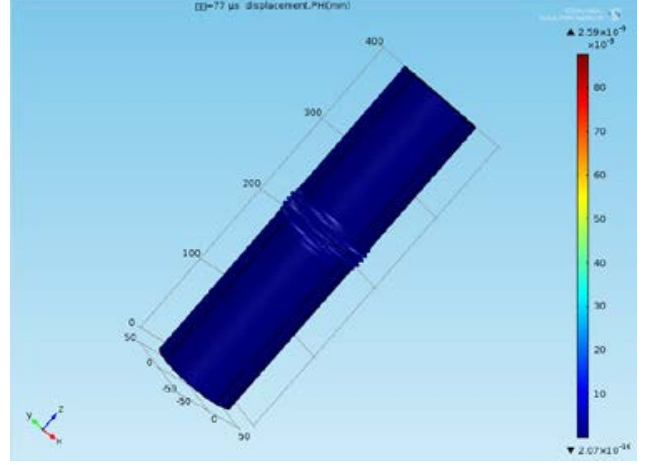

Fig.6 Propagation of $\mathrm{T}(0,1)$ mode guided wave

B. Detection Simulation of Defected Barrels

After the comparison of $\mathrm{L}$ mode and $\mathrm{T}$ mode, this paper selects $\mathrm{L}(0,2)$ longitudinal modes to detect the barrels with asymmetric defects on their inner surface. The guided wave propagation characteristics of a particular point on the barrel are shown in the Fig.7.

By setting an observation point on the surface of non-incentive end, we derive the above chart. We excite the $\mathrm{L}(0,2)$ mode guided wave according to border axial incentive method. The first waveform in the above chart is the expected $\mathrm{L}(0,2)$ mode. As can be seen from the chart, the start-up point of a waveform is about 75 us. If the propagation distance is $400 \mathrm{~mm}$, we can obtain the group velocity of $L(0,2)$ mode as follows:

$$
\mathrm{C}^{\prime}{ }_{1}=\mathrm{s} / \mathrm{t}=400(\mathrm{~mm}) / 75 \text { (us) }=5333.3 \mathrm{~m} / \mathrm{s}
$$

As can be seen from the graph of the model dispersive body tube, when the frequency is $250 \mathrm{KHz}$, the group velocity of $\mathrm{L}(0,2)$ "Cg1" equals to $5388.5 \mathrm{~m} / \mathrm{s}$, the error rate is about 1 percent. Thus the feasibility of the simulation is verified. 
Fig.7 fully shows that mode conversion happens to $\mathrm{L}(0,2)$ mode guided wave when it has asymmetric defect on the outer surface. Since the position of the defect is in the middle of the barrel, so the second group velocity, $\mathrm{C}^{\prime} 2$, of the waveform equals to $2456.4 \mathrm{~m} / \mathrm{s}$ by using the same method. By comparing this and the dispersion curves, it can be found that when the excitation frequency is of $250 \mathrm{KHz}$, the group velocity of L $(0,1)$ mode guided wave $2478.8 \mathrm{~m} / \mathrm{s}$.

In the middle of the barrel wall at $200 \mathrm{~mm}$, we set one point as the observation point, the waveforms derived are shown in Fig.8:

We can draw that the time-difference of adjacent waveforms is about 74.5us. By using the similar method, we can calculate the group velocity of $\mathrm{L}(0,2)$ mode guided wave as follows:

$$
\mathrm{C}_{3}^{\prime}=\mathrm{s} / \mathrm{t}=400(\mathrm{~mm}) / 74.5(\mathrm{us})=5369.1 \mathrm{~m} / \mathrm{s}
$$

The difference rate between experimental and theoretical results is within $1 \%$. This clearly reflects the laws of guided wave propagation process [6].

We use $\mathrm{L}(0,2)$ mode guided wave to detect barrels with asymmetric defects in the inner surface. When " $t$ " equals to 66us, the propagation characteristics of the guided wave in the barrel is shown in the Fig.9.

In the right side of the above graph, different colors of the color bar represent different displacement size. From the graph, we can see obviously that when $\mathrm{L}(0,20)$ mode guided wave passes through asymmetric defects on the outer surface, it is largely transmitted as $\mathrm{L}(0,1)$ and $\mathrm{L}(0,2)$ modes, and a small part is converted into $\mathrm{L}(0,1)$ and $\mathrm{L}(0,2)$ modes through reflection. When the excitation frequency is at $250 \mathrm{KHz}$, the group velocity of $\mathrm{L}(0,2)$ mode is as twice as that of $\mathrm{L}(0,1)$, therefore, $\mathrm{L}(0,2)$ mode is always more advanced than $\mathrm{L}(0,1)$ mode. Thus the correctness of the theory is verified by the simulation [7].

\section{Summary}

(1) Using the finite element simulation software Comsol to simulate $\mathrm{T}$ in $\mathrm{L}$ mode guided waves in barrels. Its propagation characteristics and displacement can be seen clearly. And we compare the theoretical calculation results to verify the feasibility of the simulation.

(2) On the basis of the simulation $L(0,2)$ mode guided wave propagation characteristics in the body tube, this paper studies the guided waves propagation process when there are defects in the inner surface of asymmetrical barrel. We found that $L(0,2)$ mode largely transmitted as $L(0,2)$ and $\mathrm{L}(0,1)$ guided wave mode and that a small part reflected as $\mathrm{L}(0,2)$ and $\mathrm{L}(0,1)$ mode guided wave.

\section{Acknowledgment}

It is a project supported by the National Natural Science Fund of China(No.11274199). The corresponding author is Jin Zhang.

\section{References}

[1] Wu Lin. Research on simulation methods of ultrasonic wave propagration characteristics in rail[D].Beijing Jiaotong University.2013.

[2] Sun Xuewei. Research on Propagation of Guided Waves in Complex Structures and Its Application for Damage Detection [D]. Shanghai Jiaotong University.2013.

[3] HE Cun-fu, LI Long-tao, WU Bin. Numerical Simulation of Guided Ultrasonic Wave Transmitted in Pipe[J].Journal of Beijing University of Technology, 2004,30(2):129-133.

[4] HUANG Song-ling, WANG Kun. Theory and Application of Electromagnetic Ultrasonic Guided Wave[M].Beijing: Qinghua University Press, 2013.

[5] J. Mu, L. Zhang, J.L. Rose. Defect circumferential sizing by using long range ultrasonic Guided wave[J]. Nondestructive Testing and Evaluation, 2007.22(4):239-253. 
[6] Nilesh Korde, Tribikram Kundu. Material hardness and ageing measurement using guided ultrasonic waves[J]. Ultrasonics 53 (2013) 506-510.

[7] Ming-fang Zheng, Chao Lu, Guo-zhu Chen, Ping Men. Modeling Three-dimensional Ultrasonic Guided Wave Propagation and Scattering in Circular Cylindrical Structures using Finite Element Approach. Physics Procedia 22 (2011) 112 - 118.

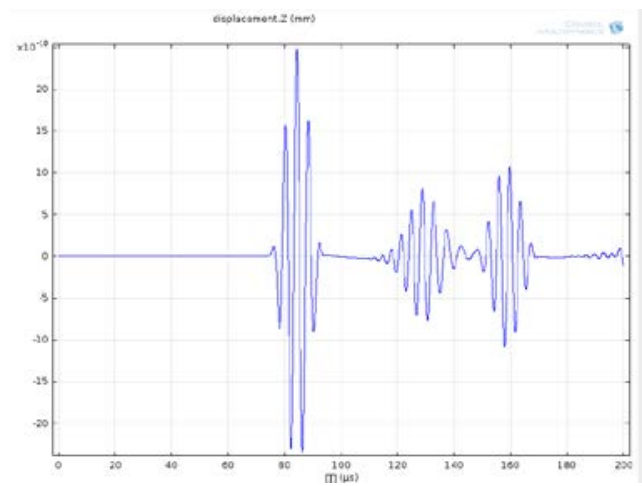

Fig.7 Observation point's displace in defective barrel

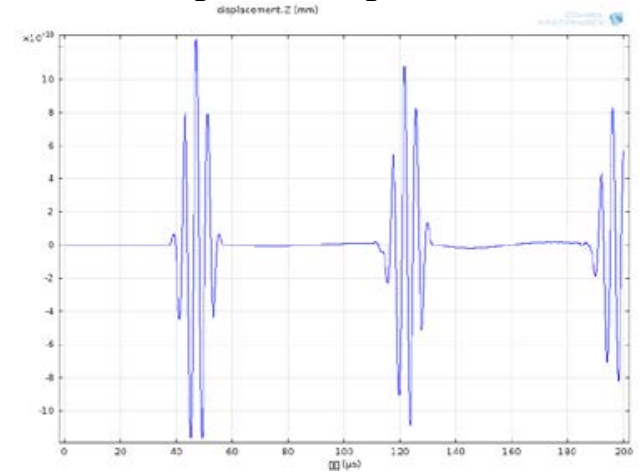

Fig.8 Displacement of defected barrel in the distance of 200mm

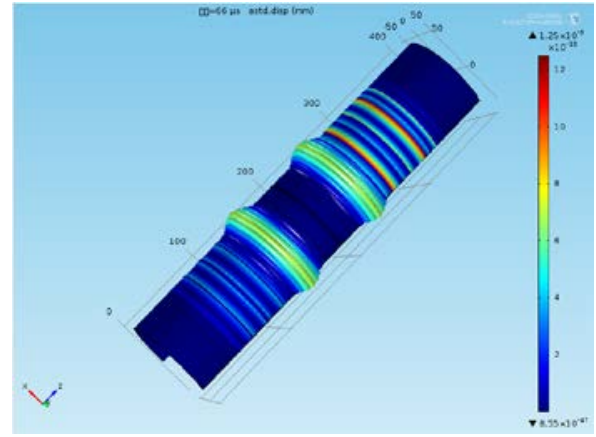

Fig.9 Propagation of $\mathrm{L}(0,2)$ mode guided wave 